\title{
Study of polymer adhesion to steel
}

\author{
Viktor Artiukh ${ }^{1,}$, Vladlen Mazur ${ }^{2}$, Volodymyr Kukhar $^{3}$, Vladimir Vershinin ${ }^{4}$, and Nikolay \\ Shulzhenko ${ }^{5}$ \\ ${ }^{1}$ Peter the Great St. Petersburg Polytechnic University, St. Petersburg, 195251, Russia \\ ${ }^{2}$ LLC ‘Saint-Petersburg Electrotechnical Company', Saint-Petersburg, 196603, Russia \\ ${ }^{3}$ Pryazovskyi State Technical University, Mariupol, 87500, Ukraine \\ ${ }^{4}$ Moscow State University of Civil Engineering, Yaroslavskoe shosse, 26, Moscow, 129337, Russia \\ ${ }^{5}$ Tula state University, 92, Lenina str., Tula, 300012, Russia
}

\begin{abstract}
Tests on the dependence of glues shear strength on: gluing temperature, keeping time before testing, cooling environment, surface roughness, surface conditions, temperature and time of drying on air and in drying oven were carried out to select proper thermoplastics to use as glues for connection of steel elements together. The highest shear strength was showed by the following materials: desmopan 150 , antifriction polyamide PA 610-L-DM, impact-resistant glass-filled polyamide. Given results allow significantly expanding scope of application of polymers as gluing compositions.
\end{abstract}

\section{Introduction}

Polymers and elastomers are increasingly used in general mechanical engineering, transport and metallurgical equipment. One of directions of polymers widespread introduction is reduction of non-technological loads in metallurgical equipment [1-5]. Polymers and elastomers are low-modulus but rather durable materials. Their energy efficiency is comparable to energy efficiency of spring grades of steel. Elastomer-based shock absorbers are simple in design and operation, they are superior to springs in many basic parameters [6-12].

On the basis of modern structural polyurethanes effective elastic couplings and shafts can be created [13]. The main difficulty is the method of connecting elastic polyurethane element with steel coupling halves, i.e. compound elastomer with steel [14-18]. Such connection should be durable and technological.

Existing glue compositions do not provide either required strength or technological ability of process of gluing. Shear strength of glue line must be not less than strength of base material (polyurethane or some other elastomer). At the same time, ultimate shear strength should be $\tau_{\mathrm{sh}}=20 \ldots 30 \mathrm{MPa}$.

Works [19-21] on study of glued connections are known for the above mentioned purposes. However, in these works required results were not achieved. In this paper polymer melts were tested as glues. Gluing technique was initially technological and required strength was achieved in stages by varying gluing conditions.

\footnotetext{
*Corresponding author: artiukh@mail.ru
} 


\section{Research Material}

To select the most promising thermoplastics as glues (refer to fig. 1) rapid tests with following polymers were carried out (given grades are used in CIS countries):

- desmopan 150;

- glass-reinforced polyethylene terephthalate PETF(B)-KC-40;

- composition based on polyamide PA 610-L-T20;

- glass-reinforced polypropylene PP-KC 30;

- glass-filled antifriction polyamide PA66-KC(M);

- antifriction composition based on polyamide (PA6-MoS2);

- composition of polypropylene with mineral filler PP-T20;

- glue-melt polyamide;

- antifriction polyamide PA 610-L-DM;

- impact-resistant glass-filled polyamide.

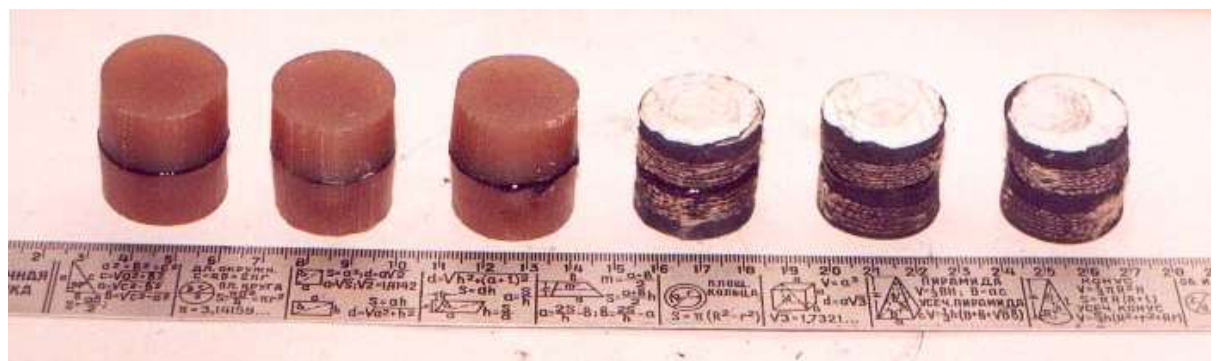

Fig. 1. Glued elastomeric samples.

Experiments showed that the highest shear strength was shown by materials: desmopan $150 \quad\left(\tau_{\mathrm{sh}}=10.5 \ldots 21.5 \mathrm{MPa}\right) ; \quad$ antifriction polyamide PA 610-L-DM $\left(\tau_{\mathrm{sh}}=29.7 \ldots 44.6 \mathrm{MPa}\right)$; impact-resistant glass-filled polyamide $\left(\tau_{\mathrm{sh}}=21 \ldots 31.7 \mathrm{MPa}\right)$. It was further decided to develop gluing technology on these materials as the most promising ones. Testing device, samples, testing machine are similar to those used in [21] (refer to fig. 2). The samples had diameter equal to $\mathrm{d}=30 \mathrm{~mm}$.

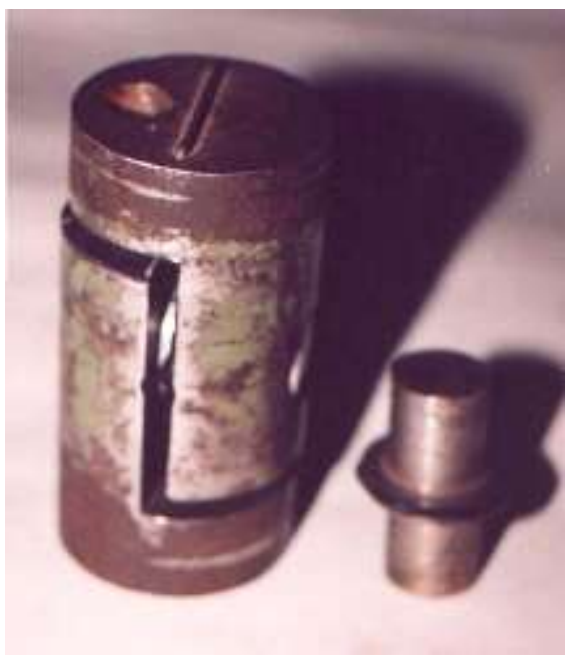

Fig. 2. Testing device and glued sample. 
Samples are made from steel grade 45 (grade is used in CIS countries, refer to fig. 3), they were heated in a special furnace; polymer granules were placed on one of heated halves of the sample and after they were melted (formation of transparent drop), this half was combined with the other one that was also heated. Then the sample was cooled on air or in water.

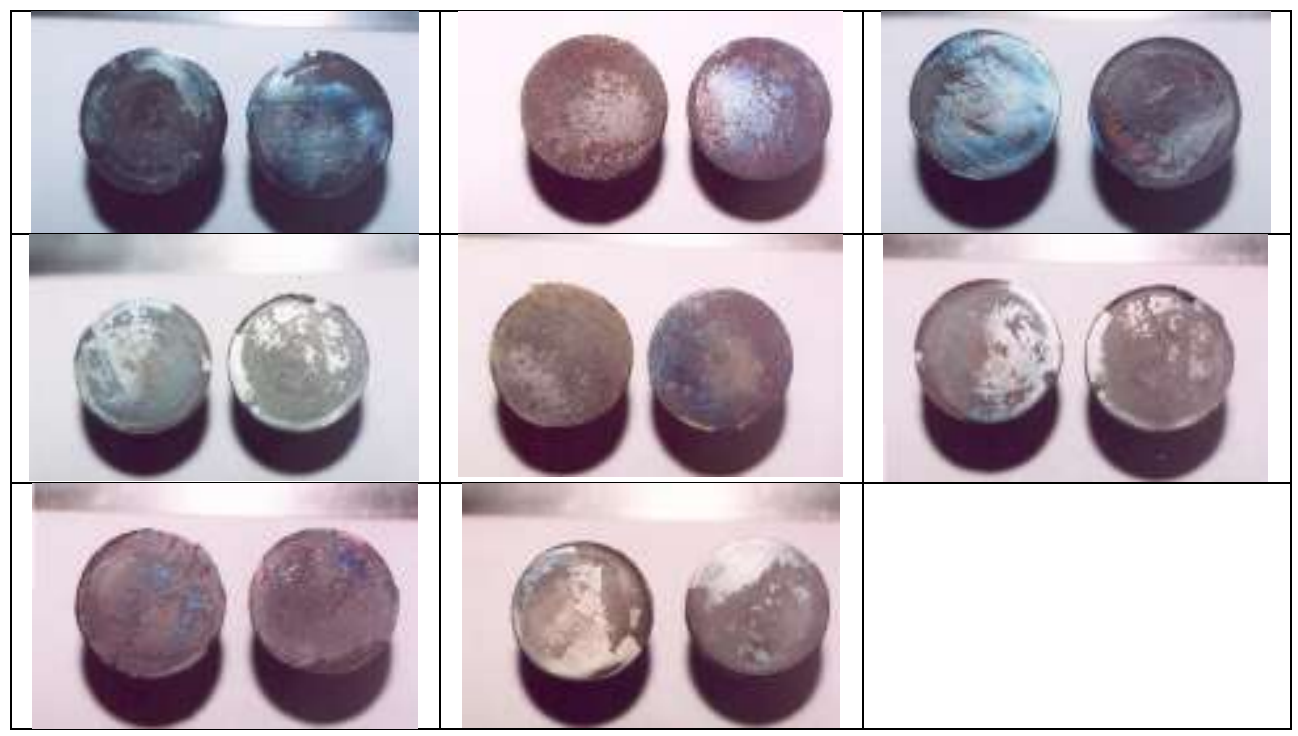

Fig. 3. End surfaces of tested samples.

Table 1 shows results of testing samples on machine IM-4A. Samples were glued together at different temperatures (indicated in table 1). They were kept before the test within 24 hours (keeping time).

The test results showed a very high adhesion strength of thermoplastics: desmopan $\tau_{\mathrm{sh}}=28.3 \mathrm{MPa}$; polyamides $\tau_{\mathrm{sh}}=41.4 \mathrm{MPa}$ and $\tau_{\mathrm{sh}}=40.0 \mathrm{MPa}$. The greatest strength shown by individual samples reaches cohesive strength of the polymer. This strength of adhesion to steel should be considered as the ultimate. For thermoplastic desmopan ultimate strength of adhesion to steel is $\tau^{*} \approx 40 \mathrm{MPa}$. For polyamides this value should be $\tau^{*} \approx 50 \ldots 60 \mathrm{MPa}$ depending on grade of polyamide. These experiments allowed determining optimum gluing temperature for each polymer. For desmopans it turned out to be equal to $270^{\circ} \mathrm{C}$ and for polyamides it was equal to $320^{\circ} \mathrm{C}$.

In addition, effect of keeping time of the glued samples before testing the strength of the glued connection was investigated. Results of these tests are shown in table 2. Desmopan requires at least five hours to achieve maximum (calculated) strength; there was no particular difference in strength (at different keeping time) for polyamides.

Table 3 shows results of testing samples that were cooled in two ways: on air and in water. All samples showed significantly greater strength under condition of air cooling.

In the next series of experiments effect of gluing surfaces state of steel samples on strength of glued connection was investigated. Samples with grinded and rough surfaces were tested. Results of these tests are given in table 4. For all desmopan samples strength of glue seam on the grinded surfaces was greater. For polyamides, on the contrary, strength on the rough surfaces turned out to be greater by $20 \ldots 30 \%$. 
Table 1. Dependence of shear strength (MPa) on gluing temperature.

\begin{tabular}{|c|c|c|c|c|c|c|c|c|c|c|c|c|c|c|c|c|}
\hline \multirow{3}{*}{ No. } & \multicolumn{5}{|c|}{ Desmopan 150} & \multicolumn{6}{|c|}{ Antifriction polyamide PA 610-L-DM } & \multicolumn{5}{|c|}{ Impact-resistant glass-filled polyamide } \\
\hline & \multicolumn{16}{|c|}{ Temperature, ${ }^{\circ} \mathrm{C}$} \\
\hline & 240 & 270 & 285 & 300 & 340 & 255 & 270 & 285 & 300 & 320 & 340 & 270 & 285 & 300 & $\begin{array}{l}32 \\
0\end{array}$ & 340 \\
\hline 1 & $\begin{array}{l}13 . \\
1\end{array}$ & $\begin{array}{l}18 . \\
0\end{array}$ & $\begin{array}{l}14 . \\
3\end{array}$ & 10.0 & 7.8 & $\begin{array}{l}23 . \\
3\end{array}$ & $\begin{array}{l}19 . \\
1\end{array}$ & 20.4 & $\begin{array}{l}22 . \\
1\end{array}$ & $\begin{array}{l}31 . \\
2\end{array}$ & $\begin{array}{l}21 . \\
4\end{array}$ & 17.1 & 19.5 & $\begin{array}{l}32 . \\
7\end{array}$ & $\begin{array}{l}25 . \\
7\end{array}$ & 31.8 \\
\hline 2 & $\begin{array}{l}15 . \\
4\end{array}$ & $\begin{array}{l}19 . \\
3\end{array}$ & $\begin{array}{l}18 . \\
0\end{array}$ & 14.0 & 8.8 & $\begin{array}{l}27 . \\
0\end{array}$ & $\begin{array}{l}23 . \\
1\end{array}$ & 22.4 & $\begin{array}{l}23 . \\
0\end{array}$ & $\begin{array}{l}33 . \\
4\end{array}$ & $\begin{array}{l}23 . \\
9\end{array}$ & 20.1 & 24.7 & $\begin{array}{l}33 . \\
1\end{array}$ & $\begin{array}{l}35 . \\
8\end{array}$ & 32.9 \\
\hline 3 & $\begin{array}{l}17 . \\
3\end{array}$ & $\begin{array}{l}23 . \\
6\end{array}$ & $\begin{array}{l}18 . \\
5\end{array}$ & 15.0 & 9.7 & $\begin{array}{l}30 . \\
5\end{array}$ & $\begin{array}{l}31 . \\
5\end{array}$ & 23.2 & $\begin{array}{l}36 . \\
7\end{array}$ & $\begin{array}{l}34 . \\
8\end{array}$ & $\begin{array}{l}27 . \\
5\end{array}$ & 24.4 & 26.5 & $\begin{array}{l}33 . \\
7\end{array}$ & $\begin{array}{l}36 . \\
6\end{array}$ & 33.5 \\
\hline 4 & $\begin{array}{l}21 . \\
8\end{array}$ & $\begin{array}{l}23 . \\
7\end{array}$ & $\begin{array}{l}19 . \\
3\end{array}$ & 15.7 & $\begin{array}{l}10 . \\
4\end{array}$ & $\begin{array}{l}31 . \\
5\end{array}$ & $\begin{array}{l}32 . \\
4\end{array}$ & 23.2 & $\begin{array}{l}36 . \\
8\end{array}$ & $\begin{array}{l}38 . \\
3\end{array}$ & $\begin{array}{l}35 . \\
4\end{array}$ & 27.5 & 33.5 & $\begin{array}{l}36 . \\
2\end{array}$ & $\begin{array}{l}39 . \\
7\end{array}$ & 35.5 \\
\hline 5 & $\begin{array}{l}22 . \\
4\end{array}$ & $\begin{array}{l}27 . \\
1\end{array}$ & $\begin{array}{l}20 . \\
0\end{array}$ & 17.0 & $\begin{array}{l}10 . \\
8\end{array}$ & $\begin{array}{l}33 . \\
2\end{array}$ & $\begin{array}{l}33 . \\
7\end{array}$ & 28.2 & $\begin{array}{l}37 . \\
0\end{array}$ & $\begin{array}{l}42 . \\
3\end{array}$ & $\begin{array}{l}35 . \\
4\end{array}$ & 28.0 & 34.0 & $\begin{array}{l}37 . \\
5\end{array}$ & $\begin{array}{l}41 . \\
8\end{array}$ & 37.9 \\
\hline 6 & $\begin{array}{l}23 . \\
8\end{array}$ & $\begin{array}{l}29 . \\
1\end{array}$ & $\begin{array}{l}24 . \\
7\end{array}$ & 17.7 & $\begin{array}{l}12 . \\
3\end{array}$ & $\begin{array}{l}33 . \\
6\end{array}$ & $\begin{array}{l}34 . \\
8\end{array}$ & 28.4 & $\begin{array}{l}37 . \\
1\end{array}$ & $\begin{array}{l}42 . \\
7\end{array}$ & $\begin{array}{l}39 . \\
1\end{array}$ & 35.7 & 36.8 & $\begin{array}{l}38 . \\
2\end{array}$ & $\begin{array}{l}42 . \\
0\end{array}$ & 38.5 \\
\hline 7 & $\begin{array}{l}24 . \\
8\end{array}$ & $\begin{array}{l}32 . \\
7\end{array}$ & $\begin{array}{l}26 . \\
6\end{array}$ & 18.0 & $\begin{array}{l}17 . \\
5\end{array}$ & $\begin{array}{l}33 . \\
7\end{array}$ & $\begin{array}{l}38 . \\
1\end{array}$ & 30.8 & $\begin{array}{l}40 . \\
2\end{array}$ & $\begin{array}{l}44 . \\
3\end{array}$ & $\begin{array}{l}40 . \\
9\end{array}$ & 37.2 & 37.7 & $\begin{array}{l}38 . \\
4\end{array}$ & $\begin{array}{l}42 . \\
2\end{array}$ & 39.5 \\
\hline 8 & $\begin{array}{l}27 . \\
7\end{array}$ & $\begin{array}{l}34 . \\
4\end{array}$ & $\begin{array}{l}27 . \\
8\end{array}$ & 24.7 & $\begin{array}{l}19 . \\
9\end{array}$ & $\begin{array}{l}35 . \\
0\end{array}$ & $\begin{array}{l}39 . \\
0\end{array}$ & 32.3 & $\begin{array}{l}42 . \\
4\end{array}$ & $\begin{array}{l}47 . \\
4\end{array}$ & $\begin{array}{l}42 . \\
6\end{array}$ & 37.6 & 38.0 & $\begin{array}{l}38 . \\
5\end{array}$ & $\begin{array}{l}42 . \\
2\end{array}$ & 40.2 \\
\hline 9 & $\begin{array}{l}29 . \\
9\end{array}$ & $\begin{array}{l}35 . \\
5\end{array}$ & $\begin{array}{l}32 . \\
0\end{array}$ & 26.8 & $\begin{array}{l}21 . \\
0\end{array}$ & $\begin{array}{l}35 . \\
8\end{array}$ & $\begin{array}{l}49 . \\
7\end{array}$ & 35.5 & $\begin{array}{l}46 . \\
4\end{array}$ & $\begin{array}{l}47 . \\
8\end{array}$ & $\begin{array}{l}44 . \\
7\end{array}$ & 39.3 & 39.5 & $\begin{array}{l}39 . \\
7\end{array}$ & $\begin{array}{l}45 . \\
4\end{array}$ & 40.8 \\
\hline 10 & $\begin{array}{l}36 . \\
5\end{array}$ & $\begin{array}{l}39 . \\
4\end{array}$ & $\begin{array}{l}32 . \\
3\end{array}$ & 27.8 & $\begin{array}{l}21 . \\
8\end{array}$ & $\begin{array}{l}51 . \\
8\end{array}$ & $\begin{array}{l}49 . \\
7\end{array}$ & 37.1 & $\begin{array}{l}47 . \\
2\end{array}$ & $\begin{array}{l}52 . \\
1\end{array}$ & $\begin{array}{l}46 . \\
1\end{array}$ & 45.1 & 43.7 & $\begin{array}{l}41 . \\
4\end{array}$ & $\begin{array}{l}48 . \\
2\end{array}$ & 41.2 \\
\hline $\begin{array}{c}\text { Averag } \\
\mathrm{e}\end{array}$ & $\begin{array}{l}23 . \\
3\end{array}$ & $\begin{array}{l}28 . \\
3\end{array}$ & $\begin{array}{l}23 \\
4\end{array}$ & 18.7 & $\begin{array}{l}14 . \\
0\end{array}$ & $\begin{array}{l}33 . \\
5\end{array}$ & $\begin{array}{l}35 . \\
1\end{array}$ & 28.2 & $\begin{array}{l}36 . \\
9\end{array}$ & $\begin{array}{l}41 . \\
4\end{array}$ & $\begin{array}{l}35 . \\
7\end{array}$ & 31.2 & 33.4 & $\begin{array}{l}36 . \\
9\end{array}$ & $\begin{array}{l}40 . \\
0\end{array}$ & 37.2 \\
\hline
\end{tabular}


Table 2. Dependence of shear strength (MPa) on keeping time before testing.

\begin{tabular}{|c|c|c|c|c|c|c|c|c|c|}
\hline \multirow{2}{*}{ No. } & \multicolumn{3}{|c|}{ Desmopan 150 } & \multicolumn{3}{c|}{$\begin{array}{c}\text { Antifriction polyamide PA } \\
\text { 610-L-DM }\end{array}$} & \multicolumn{3}{|c|}{$\begin{array}{c}\text { Impact-resistant glass- } \\
\text { filled polyamide }\end{array}$} \\
\cline { 2 - 10 } & $2 \mathrm{~h}$ & $5 \mathrm{~h}$ & $24 \mathrm{~h}$ & $2 \mathrm{~h}$ & $5 \mathrm{~h}$ & $24 \mathrm{~h}$ & $2 \mathrm{~h}$ & $5 \mathrm{~h}$ & $24 \mathrm{~h}$ \\
\hline 1 & 15.8 & 17.5 & 18.0 & 20.5 & 25.6 & 31.2 & 30.6 & 24.2 & 25.7 \\
\hline 2 & 16.8 & 24.6 & 19.3 & 31.4 & 27.1 & 33.4 & 35.0 & 30.5 & 35.8 \\
\hline 3 & 17.0 & 26.7 & 23.6 & 34.6 & 31.8 & 34.8 & 37.8 & 35.9 & 36.6 \\
\hline 4 & 17.2 & 27.8 & 23.7 & 36.1 & 35.6 & 38.3 & 39.9 & 37.2 & 39.7 \\
\hline 5 & 21.5 & 28.8 & 27.1 & 36.5 & 38.9 & 42.3 & 41.1 & 41.9 & 41.8 \\
\hline 6 & 22.0 & 29.0 & 29.1 & 36.8 & 39.8 & 42.7 & 42.1 & 42.9 & 42.0 \\
\hline 7 & 26.4 & 35.0 & 32.7 & 37.3 & 42.5 & 44.3 & 43.4 & 44.4 & 42.2 \\
\hline 8 & 28.4 & 36.1 & 34.4 & 41.4 & 43.8 & 47.4 & 44.0 & 46.0 & 42.2 \\
\hline 9 & 28.5 & 36.8 & 35.5 & 45.0 & 45.6 & 47.8 & 46.6 & 48.9 & 45.4 \\
\hline 10 & 39.1 & 39.0 & 39.4 & 47.4 & 49.5 & 52.1 & 50.6 & 49.1 & 48.2 \\
\hline Average & 23.3 & 30.1 & 28.3 & 36.7 & 38.0 & 41.4 & 41.1 & 40.1 & 40.0 \\
\hline
\end{tabular}

Table 3. Dependence of shear strength (MPa) on cooling environment.

\begin{tabular}{|c|c|c|c|c|c|c|}
\hline \multirow{2}{*}{ No. } & \multicolumn{2}{|c|}{ Desmopan 150 } & \multicolumn{2}{c|}{$\begin{array}{c}\text { Antifriction polyamide } \\
\text { PA 610-L-DM }\end{array}$} & \multicolumn{2}{c|}{$\begin{array}{c}\text { Impact-resistant glass- } \\
\text { filled polyamide }\end{array}$} \\
\cline { 2 - 7 } & Water & Air & Water & Air & Water & Air \\
\hline 1 & 12.5 & 18.0 & 13.3 & 31.2 & 25.0 & 25.7 \\
\hline 2 & 12.7 & 19.3 & 13.8 & 33.4 & 25.7 & 35.8 \\
\hline 3 & 17.8 & 23.6 & 14.4 & 34.8 & 26.0 & 36.6 \\
\hline 4 & 18.8 & 23.7 & 15.4 & 38.3 & 26.8 & 39.7 \\
\hline 5 & 22.7 & 27.1 & 16.1 & 42.3 & 27.1 & 41.8 \\
\hline 6 & 24.0 & 29.1 & 16.2 & 42.7 & 27.6 & 42.0 \\
\hline 7 & 24.4 & 32.7 & 16.5 & 44.3 & 28.8 & 42.2 \\
\hline 8 & 26.2 & 34.4 & 18.2 & 47.4 & 30.8 & 42.2 \\
\hline 9 & 27.3 & 35.5 & 27.0 & 47.8 & 31.2 & 45.4 \\
\hline 10 & 32.0 & 39.4 & 32.2 & 52.1 & 32.1 & 48.2 \\
\hline Average & 21.9 & 28.3 & 18.3 & 41.4 & 28.1 & 40.0 \\
\hline
\end{tabular}

Table 4. Dependence of shear strength (MPa) on surface roughness.

\begin{tabular}{|c|c|c|c|c|c|c|}
\hline \multirow{2}{*}{ No. } & \multicolumn{2}{|c|}{ Desmopan 150 } & \multicolumn{2}{c|}{$\begin{array}{c}\text { Antifriction polya-mide } \\
\text { PA 610-L-DM }\end{array}$} & \multicolumn{2}{c|}{$\begin{array}{c}\text { Impact-resistant glass- } \\
\text { filled polyamide }\end{array}$} \\
\cline { 2 - 7 } & Grinded & Rough & Grinded & Rough & Grinded & Rough \\
\hline 1 & 18.1 & 19.9 & 22.8 & 25.0 & 27.7 & 36.4 \\
\hline 2 & 21.5 & 20.1 & 27.8 & 25.9 & 29.8 & 38.8 \\
\hline 3 & 25.7 & 21.8 & 29.1 & 27.7 & 31.5 & 40.7 \\
\hline 4 & 27.4 & 23.8 & 33.3 & 35.3 & 33.3 & 41.8 \\
\hline 5 & 27.8 & 24.3 & 36.7 & 37.1 & 35.0 & 42.2 \\
\hline 6 & 29.0 & 26.7 & 39.4 & 40.2 & 35.1 & 43.1 \\
\hline 7 & 29.2 & 28.3 & 39.9 & 42.3 & 35.8 & 43.4 \\
\hline 8 & 29.7 & 31.6 & 43.4 & 46.4 & 39.7 & 43.6 \\
\hline 9 & 33.4 & 34.7 & 45.4 & 47.5 & 39.8 & 43.8 \\
\hline 10 & 34.5 & 35.0 & 48.0 & 49.7 & 42.8 & 48.1 \\
\hline Average & 27.6 & 26.6 & 36.6 & 37.7 & 35.0 & 42.2 \\
\hline
\end{tabular}

Another series of samples was tested with various surface conditions: non-oxidized and oxidized. Adhesion to oxidized surfaces was slightly higher (refer to table 5). 
Table 5. Dependence of shear strength $(\mathrm{MPa})$ on surface conditions.

\begin{tabular}{|c|c|c|c|c|c|c|}
\hline \multirow{2}{*}{ No. } & \multicolumn{2}{|c|}{ Desmopan 150} & \multicolumn{2}{c|}{$\begin{array}{c}\text { Antifriction polya-mide } \\
\text { PA 610-L-DM }\end{array}$} & \multicolumn{2}{c|}{$\begin{array}{c}\text { Impact-resistant glass- } \\
\text { filled polyamide }\end{array}$} \\
\cline { 2 - 7 } & $\begin{array}{c}\text { Non- } \\
\text { oxidized }\end{array}$ & Oxidized & $\begin{array}{c}\text { Non- } \\
\text { oxidized }\end{array}$ & Oxidized & $\begin{array}{c}\text { Non- } \\
\text { oxidized }\end{array}$ & Oxidized \\
\hline 1 & 18.0 & 23.3 & 31.2 & 24.8 & 25.7 & 24.8 \\
\hline 2 & 19.3 & 23.6 & 33.4 & 36.8 & 35.8 & 31.7 \\
\hline 3 & 23.6 & 26.1 & 34.8 & 41.1 & 36.6 & 36.0 \\
\hline 4 & 23.7 & 29.0 & 38.3 & 42.5 & 39.7 & 37.8 \\
\hline 5 & 27.1 & 29.6 & 42.3 & 43.7 & 41.8 & 38.3 \\
\hline 6 & 29.1 & 30.5 & 42.7 & 44.4 & 42.0 & 41.1 \\
\hline 7 & 32.7 & 30.7 & 44.3 & 47.8 & 42.2 & 42.7 \\
\hline 8 & 34.4 & 30.8 & 47.4 & 47.8 & 42.2 & 48.0 \\
\hline 9 & 35.5 & 31.2 & 47.8 & 49.1 & 45.4 & 49.4 \\
\hline 10 & 39.4 & 36.0 & 52.1 & 51.1 & 48.2 & 51.4 \\
\hline Average & 28.3 & 29.0 & 41.4 & 42.9 & 40.0 & 40.1 \\
\hline
\end{tabular}

Another series of experiments was done with different drying options: drying on air at ambient temperature and drying in drying oven at different temperatures. Results of these tests are given in table. 6. This experiments confirmed high strength of the glued connections of steel parts with hot melt glues. The main test results are shown in graphs (refer to fig. 4 and fig. 5) and diagrams (refer to fig. 6-9).

Table 6. Dependence of shear strength (MPa) on temperature and time of drying on air and in drying oven.

\begin{tabular}{|c|c|c|c|c|c|c|c|c|c|}
\hline \multirow{3}{*}{ No. } & \multicolumn{3}{|c|}{ Desmopan 150} & \multicolumn{3}{c|}{$\begin{array}{c}\text { Antifriction polyamide PA } \\
610-\mathrm{L}-\mathrm{DM}\end{array}$} & \multicolumn{3}{c|}{$\begin{array}{c}\text { Impact-resistant glass- } \\
\text { filled polyamide }\end{array}$} \\
\cline { 2 - 11 } & $\begin{array}{c}24 \mathrm{~h}, \\
\text { air }\end{array}$ & $\begin{array}{c}2 \mathrm{~h}, \\
45^{\circ} \mathrm{C}\end{array}$ & $\begin{array}{c}1 \mathrm{~h}, \\
65^{\circ} \mathrm{C}\end{array}$ & $\begin{array}{c}24 \mathrm{~h}, \\
\text { air }\end{array}$ & $\begin{array}{c}24 \mathrm{~h}, \\
70^{\circ} \mathrm{C}\end{array}$ & $\begin{array}{c}24 \mathrm{~h}, \\
85^{\circ} \mathrm{C}\end{array}$ & $\begin{array}{c}24 \mathrm{~h}, \\
\text { air }\end{array}$ & $\begin{array}{c}24 \mathrm{~h}, \\
70^{\circ} \mathrm{C}\end{array}$ & $\begin{array}{c}24 \mathrm{~h}, \\
85^{\circ} \mathrm{C}\end{array}$ \\
\hline 1 & 18.0 & 19.8 & 19.8 & 31.2 & 24.6 & 21.0 & 25.7 & 34.0 & 32.5 \\
\hline 2 & 19.3 & 21.7 & 20.8 & 33.4 & 27.6 & 21.5 & 35.8 & 36.1 & 36.6 \\
\hline 3 & 23.6 & 21.7 & 21.0 & 34.8 & 29.4 & 23.6 & 36.6 & 41.0 & 36.6 \\
\hline 4 & 23.7 & 22.0 & 23.0 & 38.3 & 34.4 & 29.0 & 39.7 & 41.6 & 37.0 \\
\hline 5 & 27.1 & 24.5 & 27.0 & 42.3 & 34.8 & 29.7 & 41.8 & 42.3 & 37.1 \\
\hline 6 & 29.1 & 24.8 & 27.3 & 42.7 & 36.7 & 31.5 & 42.0 & 42.4 & 37.7 \\
\hline 7 & 32.7 & 31.1 & 28.3 & 44.3 & 38.3 & 32.7 & 42.2 & 44.3 & 39.1 \\
\hline 8 & 34.4 & 32.3 & 28.3 & 47.4 & 38.4 & 39.6 & 42.2 & 44.4 & 40.4 \\
\hline 9 & 35.5 & 36.4 & 34.1 & 47.8 & 40.4 & 42.0 & 45.4 & 45.6 & 40.8 \\
\hline 10 & 39.4 & 39.7 & 34.7 & 52.1 & 41.4 & 46.0 & 48.2 & 48.3 & 41.8 \\
\hline Average & 28.3 & 27.4 & 26.5 & 41.4 & 34.6 & 31.5 & 40.0 & 42.0 & 37.9 \\
\hline
\end{tabular}



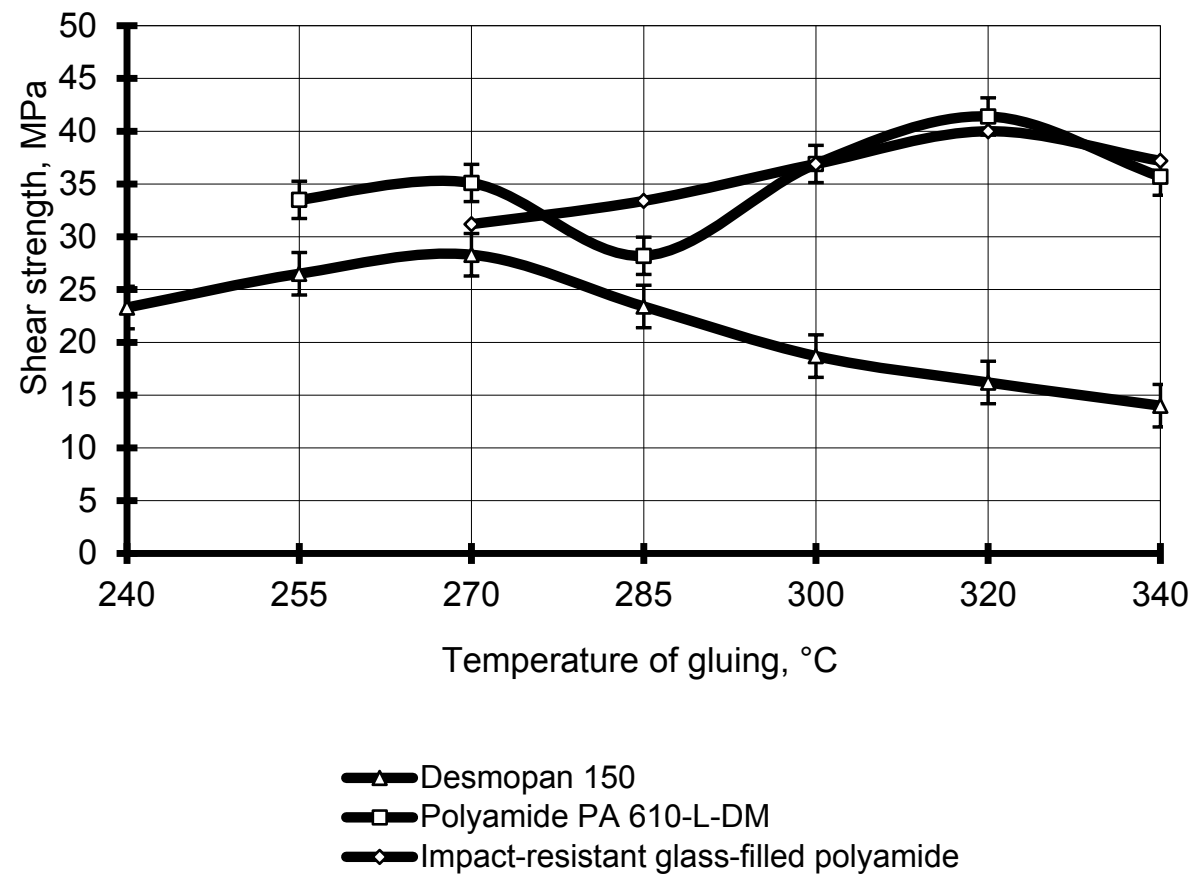

Fig. 4. Graph of relation between shear strength and temperature of gluing.

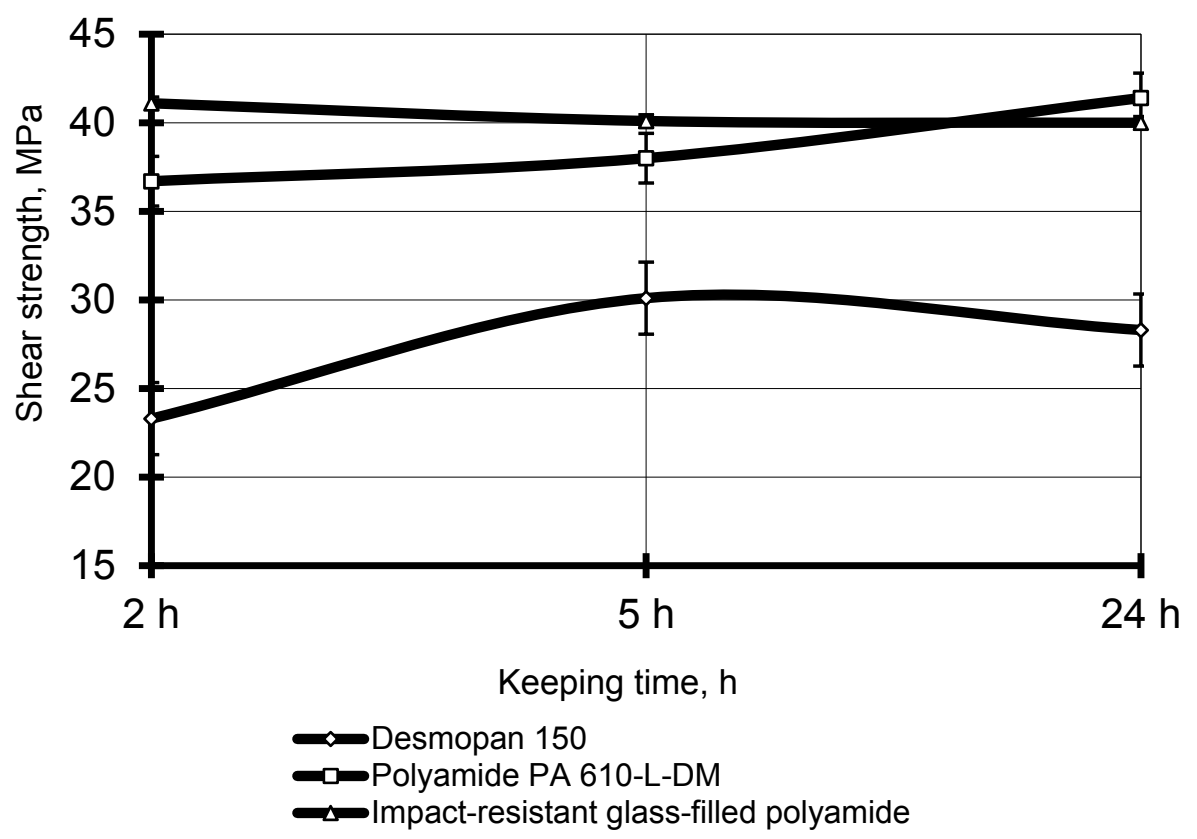

Fig. 5. Graph of relation between shear strength and keeping time before test. 


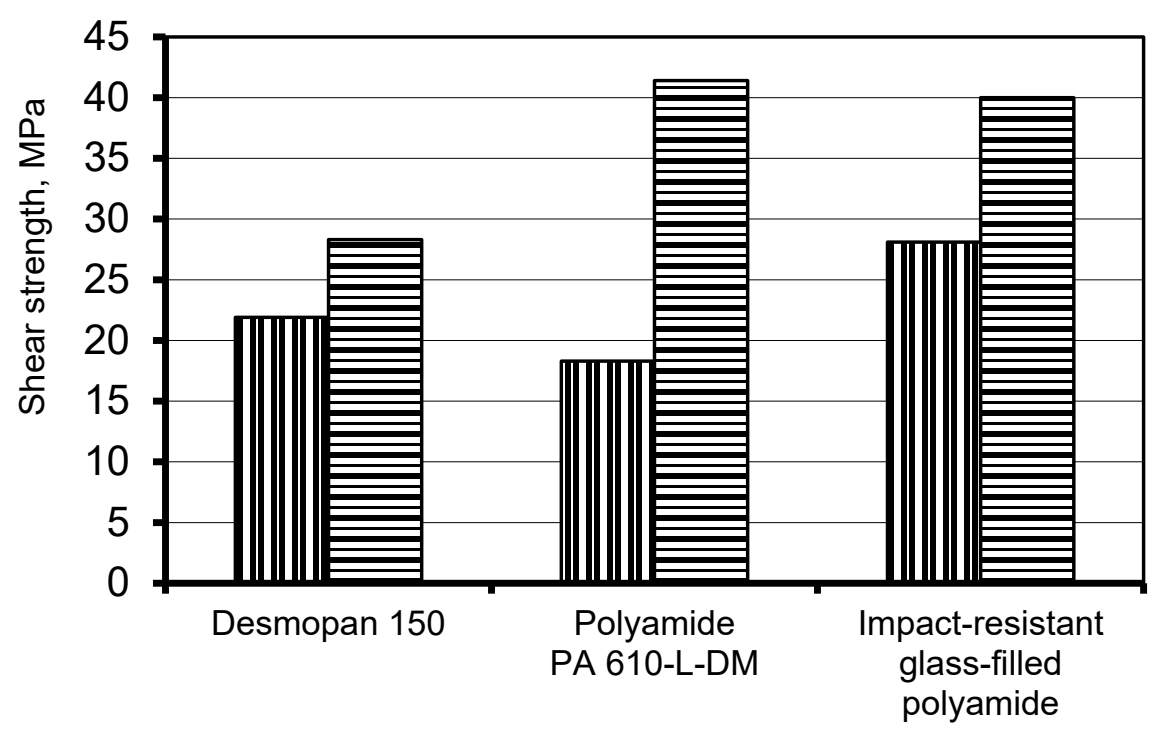

- Water $\quad$ AAir

Fig. 6. Diagram of relation between shear strength and cooling environment.

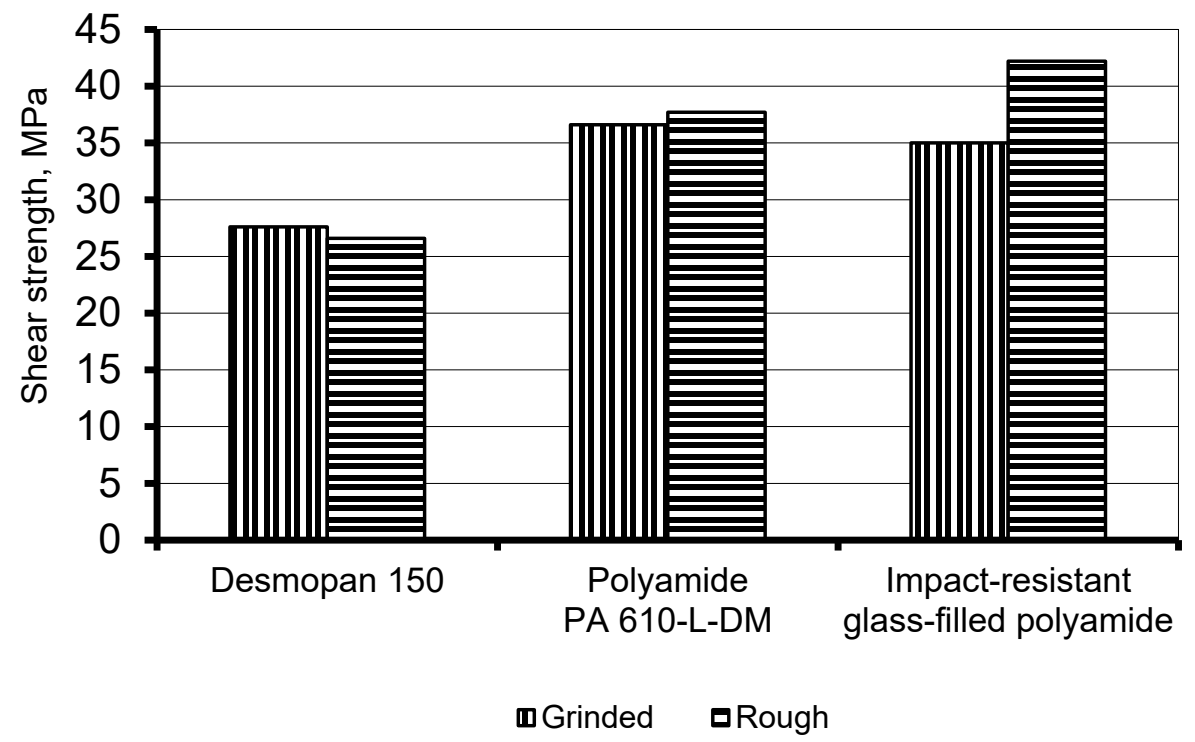

Fig. 7. Diagram of relation between shear strength and surface roughness. 


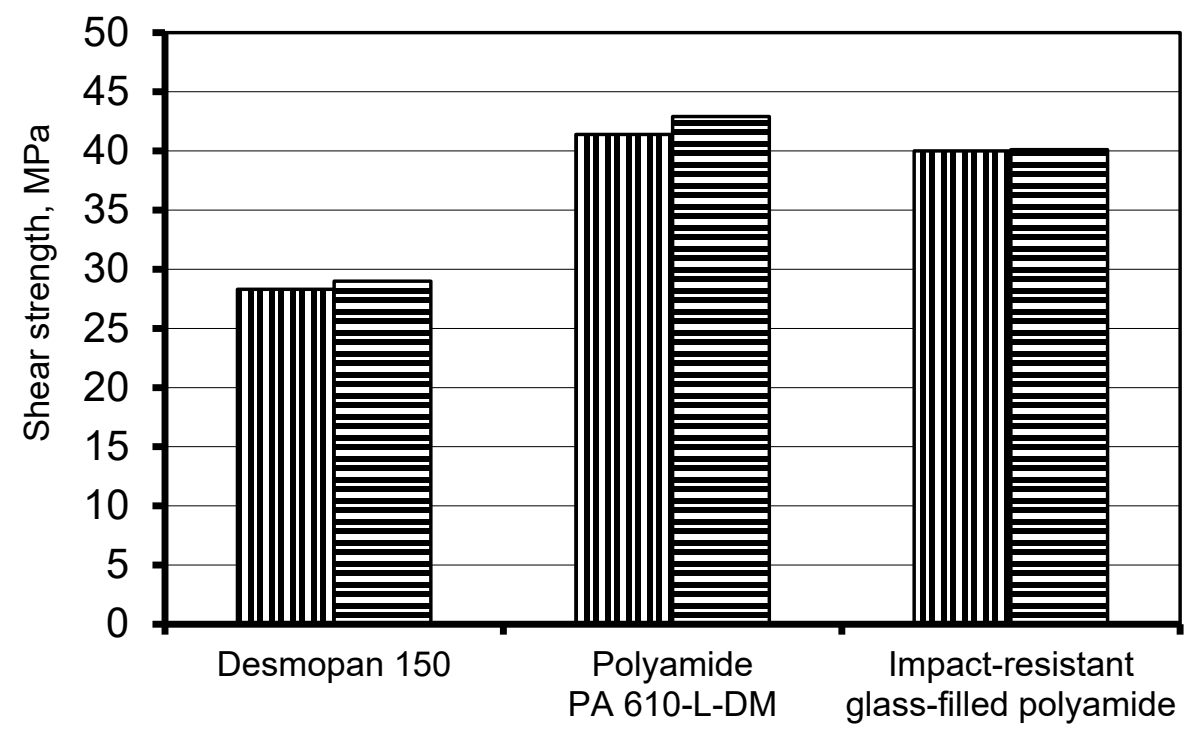

mNon-oxidized घOxidized

Fig. 8. Diagram of relation between shear strength and gluing surface conditions.

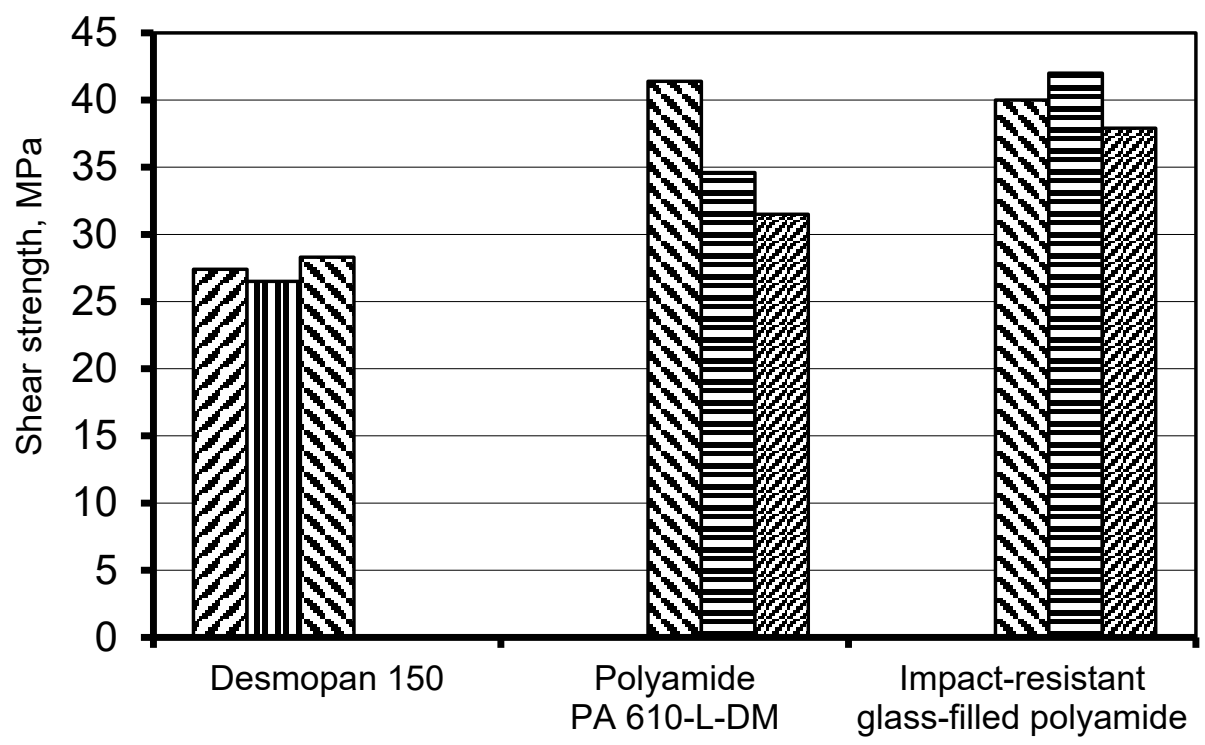

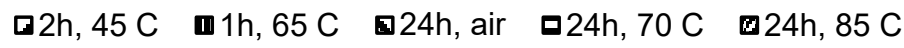

Fig. 9. Diagram of relation between shear strength, temperature and time of drying in drying oven. 


\section{Conclusions}

1. The highest shear strength showed materials: desmopan 150 , antifriction polyamide PA 610-L-DM, impact-resistant glass-filled polyamide.

2. Obtained results allow to significantly expand scope of application of polymers as gluing compositions.

3. It is necessary to conduct additional experiments to improve gluing technology.

The reported study was funded by RFBR according to the research project №19-08-01241a. The authors declare that there is no conflict of interest regarding the publication of this paper.

\section{References}

1. N. Gharaibeh, M. Matarneh, V. G. Artyukh, Loading Decrease in Metallurgical Machines. Research Journal of Applied Sciences, Engineering and Technology, 8(12), pp. 1461-1464 (2014)

2. V. Mazur, V. Artyukh, G. Artyukh, M. Takadzhi, Current Views on the Detailed Design of Heavily Loaded Components for Rolling Mills. Engineering Designer, V.37, 1, pp. 26-29 (2012)

3. Y. I. Rudaev, etc., Synergetics in mechanics of deformable body, News of KyrgyzRussian Slavic University, 10, 10, pp. 123-128 (2010)

4. V. V. Kukhar, O. V. Vasylevskyi. Experimental research of distribution of strains and stresses in work-piece at different modes of stretch-forging with rotation in combined dies, Metallurgical and Mining Industry, 3, pp. 71-78 (2014)

5. V. G. Artiukh, E. A. Galikhanova, V. M. Mazur, S. B. Kargin, Energy intensity of parts made from polyurethane elastomers. Magazine of Civil Engineering, 81(5), pp. 102-115 (2018). DOI: 10.18720/MCE.81.11

6. V. Artiukh, V. Mazur, S. Karginm, L. Zakharova, Adapters for metallurgical equipment, MATEC Web of Conferences, 170, 03028 (2018) DOI: https://doi.org/10.1051/ matecconf/201817003028

7. V. Artiukh, T. Raimberdiyev, V. Mazur, Use of CAE-Systems at Evaluation of Shock Absorbers for Metallurgical Equipment. MATEC Web of Conferences, 53, 01039 (2016) DOI: http://dx.doi.org/10.1051/matecconf/20165301039

8. M. F. Al-Quran, M. E. Matarneh, V. G. Artukh, Choice of Elastomeric Material for Buffer Devices of Metallurgical Equipment. Research Journal of Applied Sciences, Engineering and Technology, 4(11), pp. 1585-1589 (2012)

9. L. I. Ogorodov, The results of investigation of the resistance to deformations and fracture of some polymer composite materials. Mechanics of Composite Materials. V.30, 6, pp. 771-780 (1994)

10. S. N. Yakovlev, V. L. Mazurin, Vibroisolating properties of polyurethane elastomeric materials, used in construction. Magazine of Civil Engineering, 6, pp. 53-60 (2017) DOI: 10.18720/MCE.74.5.

11. I. I. Pestryakov, E. I. Gumerova, A. N. Kupchin. Assessment of efficiency of the vibration damping material «Teroson WT 129». Construction of Unique Buildings and Structures, 5(44), pp. 46-57 (2016)

12. V. G. Artiukh, S. Yu. Karlushin, E. N. Sorochan, Peculiarities of Mechanical Characteristics of Contemporary Polyurethane Elastomers. Procedia Engineering, 117, pp. 938-944 (2015) DOI: 10.1016/j.proeng.2015.08.180 
13. M. Matarneh, N. Gharaibeh, V. G. Artyukh, Effectiveness of Flexible Pin Type Couplings. International Journal of Engineering Science and Innovative Technology (IJESIT), Volume 4, Issue 2, pp. 1-7 (2015)

14. A. A. Ishchenko, etc., New technologies of revamp of metallurgical equipment by means of metal polymeric materials, Metallurgical processes and equipment, 1, pp. 55$58(2005)$

15. A. N. Levandovskiy, B. E. Melnikov, A. A. Shamkin, Modeling of porous material fracture. Magazine of Civil Engineering, 1, pp. 3-22 (2017) doi: 10.18720/ MCE.69.1

16. V. Rek, Thermal Degradation of Polyurethane Elastomers: Determination of Kinetic Parameters, Journal of Elastomers and Plastics, 35, pp. 311-323 (2003)

17. V. Rek, Kinetic Parameters Estimation for Thermal Degradation of Polyurethane Elastomers, Journal of Elastomers and Plastics, 38, pp. 105-118 (2006)

18. H. Zhang, Synthesis and Characterization of Polyurethane Elastomers, Journal of Elastomers and Plastics, 40, pp. 161-177 (2008)

19. I. A. Starostina, etc., Adhesion of polymers to metals: new approaches to assessing surface properties of metals, Glues. Sealants, Technologies, 7, pp. 27-30 (2012)

20. E. A. Galihanova, etc., Usage of technology of gluing polyurethane materials with metal surfaces, Synergy of sciences, 6, pp. 515-521 (2016)

21. A. Ishchenko, V. Artiukh, V. Mazur, S. Poberezhskii, M. Aleksandrovskiy, Experimental study of repair mixtures as glues for connecting elastomers with metals MATEC Web of Conferences, V.265, 01016 (2019) DOI: https://doi.org/10.1051/ matecconf $/ 201926501016$ 